\title{
Mariano Artigas: introducción a su obra publicada
} Mariano Artigas: Introduction to his published work

\section{JOSÉ ÁNGEL GARCÍA CUADRADO}

Universidad de Navarra

jagarcia@unav.es

Resumen. La obra publicada de Mariano Artigas (Zaragoza, 1938 - Pamplona, 2006) es muy amplia y dispersa. En este artículo se propone una posible clasificación de sus libros en cinco apartados: divulgación, manuales académicos, estudios sobre Filosofía de la naturaleza, Epistemología e Historia de la ciencia. De esta primera aproximación a su obra se extraen algunas características de su formación filosófica y su talante intelectual.

Palabras clave: Epistemología; Filosofía de la naturaleza; Filosofía de la ciencia; Historia de la ciencia; racionalidad; Popper; Galileo; darwinismo.

Abstract. The published work of Mariano Artigas (Zaragoza, 1938 - Pamplona, 2006) is extensive and addresses a very wide range of topics. The purpose of this paper is to offer a possible classification of his published books into five categories: general public, textbooks, studies on Philosophy of Nature, Epistemology and History of Science. From this first approach to his works it is possible to grasp some features of his philosophical formation and his intellectual insights.

Keywords: Epistemology; Philosophy of Nature; Philosophy of science; History of Science; rationality; Popper; Galileo; Darwinism. 
A diez años de distancia del fallecimiento del profesor Mariano Artigas parece llegado el momento de empezar a valorar su obra. Para ello se hace imprescindible conocer de manera completa su producción escrita. En este artículo se pretende llevar a cabo una primera clasificación de sus obras de modo temático, presentando en rasgos muy generales las principales características de sus escritos. En el anexo se podrá consultar la cronología de sus obras. Examinando atentamente su producción filosófica se puede decir que no hay propiamente una evolución en su pensamiento, sino más bien una profundización en sus tesis filosóficas fundamentales, que con los años va perfilando en sus contornos. Por eso, creo oportuno introducir el conjunto de su obra desde el punto de vista sistemático, más que diacrónico. De esta manera espero contribuir a facilitar los primeros pasos de cara a una futura edición de sus obras completas.

\section{Formación académica y método de trabajo}

Hasta el momento no disponemos de ninguna biografía completa de la vida de Mariano Artigas. Con motivo de su muerte aparecieron algunas semblanzas in memoriam, relativamente extensas, que pueden ser de gran utilidad para adentrarse en su obra (García Cuadrado 2007, Collado 2008).

Un primer aspecto a considerar es su formación académica que dejará una impronta indeleble en todos sus escritos. Poseía una formación científica muy sólida. Completó la licenciatura en Ciencias Físicas en la Universidad de Barcelona (1960) y años más tarde (1968) se doctoró en la misma Universidad con una investigación titulada Relación y estructura en la mecánica newtoniana codirigida por Roberto Saumells, catedrático de Filosofía de la Naturaleza (López Quintás 1970, 657-670) y Luis María Garrido Arilla, catedrático de Física Matemática.

Para cuando obtuvo el doctorado en Físicas, Mariano Artigas ya era doctor en Filosofía (1963) por la Universidad Lateranense de Roma con la tesis El problema de la substancialidad de las partículas elementales: estudio sobre la aplicabilidad de la noción de substancia a la microfísica. Esta tesis fue dirigida por el profesor Roberto Masi, un conocido exponente de la 
escolástica romana del momento. En su trabajo, Artigas busca una línea de diálogo entre la filosofía clásica y la ciencia moderna, a propósito de la noción clásica de sustancia, rica en doctrina metafísica.

Su formación filosófica se completó con su doctorado civil en la Facultad de Filosofía (1979) de la Universidad de Barcelona con la tesis "La confiabilidad de la ciencia y su impacto filosófico", donde aborda el estudio de la epistemología del momento. Esta tesis fue dirigida por el catedrático Francisco Canals, bien conocido representante de la Escuela Tomista de Barcelona (Forment 1998, 439-502). Además, unos años antes (1964) Mariano Artigas había recibido la ordenación sacerdotal, lo que suponía haber terminado sus estudios filosófico-teológicos. Durante algunos años fue también profesor de Teología en Barcelona. La perspectiva teológica se encontraba siempre presente como trasfondo en su labor intelectual. De una manera u otra, su intención fue contrastar las cuestiones científicas y filosóficas con la fe cristiana; pero su talante intelectual se encontraba en las antípodas de actitudes fideístas que acaban por debilitar la razón. En efecto, la fe religiosa no es en él un cómodo expediente para sobrevolar por encima de los conflictos entre ciencia y religión. Para él, los conflictos entre fe cristiana y ciencias experimentales debían ser acometidos con extremado rigor, tanto especulativo como histórico, con un respeto exquisito por la verdad. Esto le posibilitaba acercarse a los problemas con una mente abierta, sin prejuicios, abierto a reconocer los errores que una deficiente comprensión religiosa pudo hacer del dato científico; y al mismo tiempo, reconociendo las limitaciones de la ciencia cuando se arroga el privilegio de ser la instancia última del saber. El puente que hace posible el diálogo entre ciencia y fe es una razón filosófica abierta a la instancia metafísica.

En definitiva, cuando en 1980 comienza a publicar sus primeros trabajos poseía una base científica de alto nivel académico, así como una sólida formación filosófica clásica (con una fuerte impronta de la metafísica escolástica) pero abierta a la epistemología del siglo XX. Todos estos ingredientes crearon las condiciones idóneas para desarrollar una amplia y profunda actividad en los campos de Filosofía de la Naturaleza y Filosofía de la ciencia. Por otro lado, su contacto vital con la Teología le dotaba de una gran sensibilidad 
hacia las cuestiones últimas, así como hacia los temas fronterizos entre ciencia y religión. Sobre esa envidiable formación intelectual comenzaría a desarrollar su propio método de trabajo intelectual.

Un primer examen al conjunto de su obra escrita ya nos revela un modo de trabajar que se manifestó con el tiempo extraordinariamente fecundo. Gran parte de su esfuerzo consistió en la divulgación accesible, a la par que rigurosa, de los principales problemas científicos. Mariano Artigas se mantuvo al día de las principales ideas de la ciencia y de la epistemología del momento buscando hacer llegar a un público más amplio los aspectos relevantes de esas ideas para la filosofía y la religión. Y esta actitud se encontró presente también en los periodos de tiempo en los que se encontraba alejado de la actividad académica. Uno de los principales méritos de Artigas consistió en hacer accesible la ciencia del momento: esto le hizo adquirir un tono de escritura claro, directo, con comparaciones plásticas y pedagógicas: era un gran divulgador. Junto a esa facilidad para explicar los problemas profundos de manera sencilla, Artigas era también un gran lector, siempre al día de las principales obras publicadas, como lo demuestran sus numerosas reseñas y artículos divulgativos. Ese material le serviría después para redactar sus libros y artículos especializados. Sobre esa base amplia de lectura de los principales autores fue construyendo un modo de pensar propio.

Una última anotación que no pasará inadvertida a quien examine con detenimiento su curriculum vitae: especialmente en sus últimos años supo aunar esfuerzos con otros investigadores universitarios. Encontró en esta fórmula - poco frecuente entonces en la investigación humanística- un modo de trabajar con otros estudiosos de reconocido prestigio en su ámbito creando las condiciones para un enriquecimiento mutuo. Este modo de proceder manifiesta también una característica no muy frecuente en medios académicos: la capacidad de trabajar en equipo, de confrontar las propias ideas con otros planteamientos, tal vez en un principio muy alejados de los propios. Así sucedió, por ejemplo, con los libros publicados en colaboración con Thomas Glick, William Shea, o Karl Giberson, entre otros. En el fondo, en Mariano Artigas latía quizás la conciencia de la propia limitación en el saber; limitación que no le encerraba en su propio punto de vista sino 
que buscaba superarse a través del diálogo abierto dentro de la comunidad científica.

\section{Ensayo de clasificación de sus obras}

Toda clasificación implica la adopción de unos criterios hasta cierto punto arbitrarios y discutibles. La obra escrita de Mariano Artigas es extensa, lo que da idea de su gran capacidad de trabajo, teniendo en cuenta, además, que su desaparición fue relativamente prematura. Intentar clasificar su obra implica algún riesgo que conviene afrontar. Por ejemplo, la distinción entre obras de divulgación, manuales y estudios monográficos obedece a unos criterios cuyos límites no son netos. Los contenidos de unas obras y otras se solapan hasta el punto en el que en ocasiones no es fácil trazar una frontera clara entre ámbitos, pues su divulgación era rigurosa, y su investigación asequible a un público muy diversificado.

En la clasificación que ahora propongo me ceñiré fundamentalmente a sus libros publicados. Hay valiosos artículos de divulgación o publicados en revistas especializadas muy relevantes de su pensamiento, pero todos ellos vienen a confluir en algunos libros que sintetizan sus aportaciones. Por otro lado, parece evidente que los temas que trata se encuentran interrelacionados de manera muy estrecha: el análisis de la estructura del mundo material lleva a cuestiones últimas acerca de qué es la realidad, y cómo puede conocerse. En definitiva, las cuestiones de filosofía natural reclaman las cuestiones epistemológicas, donde también se hallan implicadas cuestiones acerca de la historia de la ciencia.

Teniendo en cuenta todas esas posibles dificultades me atrevo a sugerir de manera orientativa una posible división de su obra escrita en los siguientes apartados.

Obras de divulgación.

Manuales de Iniciación filosófica.

Estudios sobre filosofía de la Naturaleza.

Estudios sobre filosofía de la ciencia.

Estudios sobre historia de la ciencia. 


\section{Obras de divulgación: artículos y libros}

Cuando el profesor Artigas debía presentar su curriculum vitae dejaba constancia en un apartado de los artículos de divulgación: esta actividad no la consideraba en modo alguno un desdoro en el elenco de sus publicaciones. De hecho, son decenas los artículos divulgativos que publicó en revistas culturales y en prensa diaria. En todo caso, la mayoría de esos artículos se publicaron en revistas de limitada difusión dirigida especialmente a un público culto, aunque no especializado en cuestiones científicas. En esos artículos incidía en las cuestiones de fondo con un lenguaje claro y directo - polémico también en ocasiones- con una referencia especial a los problemas relativos a ciencia y religión.

Esos artículos de divulgación encontraban múltiples cauces de expresión: en no pocas ocasiones veían la luz en diversos medios de difusión: por ejemplo, se publicaban en castellano y en catalán, en dos revistas culturales distintas. Se trataba de cuestiones de actualidad, con motivo de un nuevo descubrimiento científico o una obra relevante para la ciencia. Con el tiempo, ese esfuerzo de lectura y divulgación de la actualidad científica cristalizó en un amplio número de artículos, que, debidamente elaborados, dieron lugar a libros de divulgación de notable éxito editorial. En las sucesivas ediciones, Artigas introducía muchas veces nuevos temas o capítulos, enriqueciendo sustancialmente su contenido. Algunos de esos títulos llegaron a incorporarse a la colección de "Iniciación filosófica" de Eunsa, a la que nos referiremos más adelante.

Su primer libro de divulgación apareció en 1984 en la Editorial Palabra (Epalsa, Madrid): llevaba por título Ciencia, razón y fe y estaba prologado por su buen amigo, el profesor Evandro Agazzi, actual Presidente de la Academia Internacional de Filosofía de la ciencia. El formato y las dimensiones del libro dejaban clara su intención de dirigirse a un público muy amplio. Los temas abordados en este primer libro son un reflejo de los temas a los que se dedicará a lo largo de su vida: evolucionismo y cristianismo, el caso Galileo, el método científico y sus límites, el diálogo entre ciencia y fe, el materialismo científico, etc. Por lo demás, el mismo título del libro llegará 
a ser muy representativo de su pensamiento: el diálogo entre la ciencia moderna y la fe cristiana (aparentemente truncado a partir de la Modernidad) se puede salvar - según Artigas - a través de una razón que no se encierra en los límites de la ciencia experimental. Pero si a la razón se le pide una rectificación crítica de sus límites, la fe no puede recluirse en un cerrado voluntarismo que renuncie a una mejor comprensión de la verdad mediante esa razón ampliada. Pasados los años, cuando Mariano Artigas promovió un Grupo de estudio interdisciplinar lo denominó precisamente así: ciencia, razón y fe (CRYF). Las ediciones de este libro se sucedieron rápidamente $(1985,1986,1992)$. En la $5^{a}$ edición (1992) añadió un apéndice poniendo al día algunas cuestiones. Poco tiempo después, la editorial Eunsa se interesó por este libro y le ofreció publicarlo en la colección de manuales de "Iniciación filosófica". Esto hizo que sometiera el libro a una revisión completa de principio a fin, incorporando además dos nuevos capítulos: uno sobre el evolucionismo y otro sobre la encíclica Fides et ratio. En 2004 apareció la $1^{\mathrm{a}}$ edición en Eunsa (la $6^{\mathrm{a}}$ en total): pronto fue reimpresa (2006) y más tarde salió, ya póstumamente, la $2^{\text {a }}$ edición en dicha editorial (2011).

Animado quizás por el éxito editorial de este primer trabajo, acometió de manera más monográfica uno de los temas ya tratados: el evolucionismo y sus implicaciones filosóficas. En 1985 publica Las fronteras del evolucionismo con el mismo formato divulgativo que el anterior. Esta vez el libro venía prologado por Sir John Eccles -Premio Nobel de Medicina en 1963- con el que Mariano Artigas mantuvo una relación personal y epistolar significativa. Además se añade como epílogo un diálogo con el mismo autor, titulado "Alma humana, ciencia y religión”. El éxito editorial fue notable. El mismo año de su publicación se editó en tres ocasiones; en 1986 salió la $4^{\text {a }}$ edición. Pasados los años, en 1992, la misma editorial volvió a editarlo con un apéndice del autor en el que se ponían al día algunas cuestiones tratadas. De esa edición actualizada se realizó la traducción al portugués y al italiano (ambas aparecieron en 1993). Sin embargo, la editorial decidió no reeditarlo. Por esta razón, el profesor Artigas pensó ofrecerlo a la editorial Eunsa para ser incluida en la colección de "Iniciación filosófica”. Después de una revisión profunda y puesta al día, adaptándose a los requerimientos 
académicos de esta colección, se publica finalmente en 2004 “una versión completamente nueva" como el mismo autor reconoce en la introducción. El libro enmarca en un amplio contexto la teoría evolutiva: el origen del universo y de la vida, el origen del hombre, pasa revista a continuación a los temas filosóficos implicados (creación, azar y finalidad, entre otros) así como a la recepción por el cristianismo de las teorías evolutivas. Artigas distingue entre los logros científicos y la interpretación, muchas veces ideologizada, del dato científico. De esta manera se facilita el diálogo riguroso y objetivo sobre el tema.

En los mismos años en los que revisaba las primeras ediciones de los dos libros anteriores, Artigas continúa su labor divulgativa con dos nuevos trabajos donde aborda de nuevo temas fronterizos entre fe y ciencia. El primero de ellos (El hombre a la luz de la ciencia, Palabra, 1992) se ocupa de la imagen del hombre que se proyecta a través de la ciencia. Por una parte, la ciencia - con su grandeza, pero también con sus limitaciones- es una tarea humana; al mismo tiempo, la ciencia estudia al hombre como un ser más en la naturaleza, lo cual entraña el riesgo de reducirlo a estructuras observables por el hombre: los problemas del alma humana, la libertad, el materialismo o la creación del universo son abordados aquí de manera sencilla pero abriendo la razón humana a perspectivas de mayor calado filosófico. En esta ocasión, el libro viene prologado por el Gran Canciller de la Universidad de Navarra y obispo prelado del Opus Dei, Mons. Álvaro del Portillo, con el que se mantiene un diálogo - publicado como apéndice bajo el título "Ciencia y conciencia”. Al igual que el profesor Artigas, Mons. del Portillo poseía una buena formación científica y una fina sensibilidad humanística. Es interesante constatar cómo en la investigación de Artigas las cuestiones científicas no se desligaban de la cuestión antropológica. La naturaleza no es simplemente un objeto de estudio ajeno al que le resulta extraña la realidad humana; por eso, en sus obras, Artigas subraya, de un modo u otro, la singularidad de la persona humana frente al cosmos (Karol 2000, 209-274).

En ese mismo año de 1992 publicó un libro de bolsillo titulado Ciencia y fe: nuevas perspectivas (Eunsa). Se trata de un texto dirigido a un amplio público, sencillo, claro, directo; pero escrito por un hombre de ciencia. Se 
puede decir que el interés es más bien apologético (en el sentido noble del término): dirigido a un cristiano de finales del siglo XX que se mueve en un mundo dominado por la visión científica del mundo. Artigas da argumentos para comprender de modo sencillo los alcances y los límites de la ciencia experimental, y cómo esto afecta a las grandes cuestiones teológicas, antropológicas y éticas. A pesar de que los temas tratados resultan en su mayoría familiares, Artigas afirma en la "Presentación" que han sido redactados completamente de nuevo. De entre ellos, el capítulo dedicado a la nueva cosmovisión es el que refleja mejor la paradoja de la ciencia moderna: una ciencia sin metafísica intenta autoexplicarse sin Dios; pero esa actitud ya es de algún modo metafísica.

Con este libro se puede decir que culmina una primera fase de su obra más centrada en la divulgación. Sin embargo, esa preocupación estuvo presente hasta el final de su vida. Vuelve sobre el tema de la evolución en su libro póstumo (apareció en 2007, pocos meses después de su muerte): Origen del hombre. Ciencia, Filosofía y Religión, escrito en colaboración con Daniel Turbón, catedrático de Antropología Física de la Universidad de Barcelona y experto en Evolución humana. El formato del libro es divulgativo, pero se trata de una puesta al día de cuestiones sometidas continuamente a revisión por nuevos hallazgos o hipótesis. Junto a temas ya tratados con anterioridad, pero con una perspectiva más actual y con abundancia de datos científicos, los autores abordan, entre otras cuestiones, el Diseño inteligente, con sus luces y sus sombras, subrayando también la necesidad de distinguir la ciencia de las interpretaciones ideologizadas de la misma. El libro contó con tres ediciones en el mismo año 2008.

Finalmente hay que reseñar el diccionario aparecido en 2007: Ciencia y religión. Conceptos fundamentales. La editorial Eunsa animó al profesor Artigas a reelaborar en forma de diccionario los grandes temas sobre Ciencia y religión que ya conocía muy bien, después de toda una vida dedicada a ellos. El resultado final es un elenco de veinticinco voces representativas de los principales problemas surgidos en la historia acerca de las relaciones entre ciencia y religión. La extensión de las voces y el lenguaje son asequibles a todo tipo de lector. A pesar de ser voces que pueden ser consultadas de 
manera independiente, la idea que vertebra el trabajo es la búsqueda de una imagen unitaria del mundo y de nosotros mismos; y en esa búsqueda, ciencia y religión nos ofrecen perspectivas complementarias, si somos capaces de superar viejos clichés cientificistas.

\section{Manuales de filosofía}

En 1981 la Facultad de Teología de la Universidad de Navarra comenzó a impartir los cursos institucionales (bachillerato teológico) que incluían dos cursos filosóficos previos a los estudios estrictamente teológicos. Pronto se vio la necesidad de manuales adecuados para los alumnos que comenzaban esos estudios con una orientación teológica. De este modo, en 1982, nació la colección "Iniciación filosófica" en el seno de la editorial Eunsa, ligada a la Universidad de Navarra. A Mariano Artigas le encargaron dos manuales en esta colección, que aparecieron casi simultáneamente en 1984.

El primer texto era el de Filosofía de la naturaleza que fue redactado conjuntamente con el profesor Juan José Sanguineti. Ambos se conocieron en Roma y compartían intereses filosóficos; de hecho habían elaborado algunos materiales didácticos para la enseñanza de la Filosofía de la Naturaleza que sirvieron de base para este libro. El planteamiento de este manual era fundamentalmente clásico, muy ligado a la cosmología escolástica. De este modo se exponía lo relativo al ente móvil, el hilemorfismo, la sustancia material, y los accidentes sensibles (lugar, espacio, tiempo, cantidad) así como las cualidades corpóreas. Con el paso del tiempo, este enfoque irá siendo puesto al día de manera paulatina: de hecho, fue quizás el texto más reelaborado por Artigas en sus sucesivas reediciones. La $2^{\mathrm{a}}$ edición es de 1989, y ya aparecía corregida y notablemente aumentada, con algunas cuestiones particulares sobre las interpretaciones contemporáneas y el origen del universo. Sobre este texto se realizó la edición italiana, prologada también por Evandro Agazzi. En 1993 apareció la 3a edición castellana, nuevamente revisada y actualizada. La siguiente edición -la $4^{\mathrm{a}}$ - apareció cinco años más tarde (1998). En ella Artigas aparece ya como autor único, con un texto completamente renovado. En el prólogo, el profesor Sanguineti 
explica que el objetivo de esta nueva edición - muy renovada con respecto a la $1^{\text {a }}$ - es "dar una visión filosófica de fondo de las realidades naturales del mundo material que concilia aspectos perennes del planteamiento metafísico clásico con la nueva cosmovisión de la naturaleza que emerge de la ciencia moderna" (Sanguineti 2003, 18). El esfuerzo por repensar la naturaleza descubriendo en ella una visión unitaria pasa por un diálogo - a veces complejo, pero fecundo a la postre-, entre la metafísica clásica y la ciencia moderna: esta será una de las líneas de fuerza de todo el pensamiento de Artigas. Este texto ofrece una filosofía de la naturaleza puesta al día y se sigue utilizando como manual en muchos centros filosóficos y teológicos. Desde entonces han aparecido dos reimpresiones (2003, 2008), y una traducción al portugués (2005).

Una suerte muy distinta corrió el otro manual de la colección de "Iniciación filosófica”: Introducción a la filosofía (Eunsa, 1984). Era un texto breve, sencillo, donde se abordaban de manera sucinta las cuestiones básicas: naturaleza de la Filosofía; división de la misma, relaciones entre Filosofía y cristianismo, etc. Las fuentes para su redacción bebían fundamentalmente de la tradición aristotélico-tomista. La utilidad de este manual viene avalada por las numerosas ediciones que contó en los años sucesivos: cinco en total (1984, 1987, 1990, 1995, 1997); así como una traducción al inglés (1990). Poco después apareció la encíclica Fides et ratio y la editorial pensó en ofrecer una versión actualizada de esta materia, lo que exigía una reelaboración más completa del manual que desde su $1^{\mathrm{a}}$ edición se mantenía inalterada. Artigas había canalizado sus intereses en otra dirección y declinó el encargo de revisión del texto. Este título de la colección se confió al profesor Tomás Melendo: en 2001 apareció el nuevo manual que sustituiría al más básico redactado por Mariano Artigas.

Por aquel entonces, ya había comenzado a trabajar en un nuevo manual para la colección de "Iniciación filosófica": Filosofía de la ciencia (1999). La escasez de textos adecuados de esta materia -relativamente reciente pero de importancia creciente en los estudios filosóficos- animó a la editorial a proponer al profesor Artigas la redacción de este manual. Ya por entonces había escrito mucho acerca del tema, principalmente desde el punto de vista 
histórico. En este manual realiza una óptima sistematización ofreciendo un curso completo, claro y profundo sobre la filosofía de la ciencia. En estas páginas aborda el impacto de las ciencias en la cultura actual, proponiendo un desarrollo histórico muy ajustado, para ofrecer a continuación una reflexión filosófica sobre la ciencia, de la mano de la epistemología contemporánea, y sus críticas. Con este bagaje aborda la naturaleza de las ciencias, su unidad y diversidad, e intenta su integración armónica con los demás saberes humanos. Finalmente, aborda la cuestión del método y el valor de la ciencia. El texto, muy elaborado, contó con una buena acogida: ha sido reimpreso en dos ocasiones (2006, 2009).

De modo general se podría decir que los primeros años de actividad de Artigas como escritor estuvo muy marcada por la preocupación divulgativa y didáctica, desde su rigurosa formación científica y filosófica. No abandonó nunca esta preocupación mientras abordaba con estudios y trabajos más especializados algunas cuestiones que caracterizarán más su pensamiento.

\section{Estudios sobre Filosofía de la Naturaleza}

En 1987 apareció en forma de separata El problema de la substancialidad de las partículas elementales. Se trata de un extracto de su tesis doctoral defendida en la Facultad de Filosofía de la Pontificia Universidad Lateranense de Roma en julio de 1963. En la revisión de 1987 reconoce la validez de lo expuesto años antes, aunque con las debidas matizaciones. El objetivo del trabajo es exponer la vigencia de la categoría metafísica de substancia mostrando que no contradice las conceptualizaciones físicas modernas. Como se puede observar esta es una de las preocupaciones fundamentales de Artigas: afinar los instrumentos conceptuales de la metafísica clásica para entrar en un diálogo constructivo con la ciencia moderna.

En 1992 publica La inteligibilidad de la naturaleza (Eunsa). En la introducción, Artigas reconoce que este libro es el fruto de su enseñanza universitaria en la Universidad de Barcelona y de Navarra, a la que se acababa de incorporar como profesor de Filosofía de la Naturaleza. En este texto condensa una reflexión de más de treinta años con un trasfondo metafísico deudor de 
Aristóteles y santo Tomás, cuyas doctrinas filosóficas, una vez liberadas de explicaciones científicas ya periclitadas, mantienen su fuerza a la luz de la cosmovisión actual. También han influido en este libro algunos aspectos de la filosofía de Leibniz y Bergson, en diálogo con la epistemología del siglo XX, donde Artigas destaca las aportaciones de Popper y Agazzi. Estas ideas se articulan en torno a las nociones de dinamismo y estructuración en la naturaleza, que complementan el hilemorfismo escolástico. Se trata de una sistematización bastante original de la filosofía de la naturaleza que culmina en la persona humana. Algunas de las ideas de este libro se verán después plasmadas en su manual de Filosofía de la Naturaleza ya reseñado anteriormente, aunque ahora dirigido a un público diverso, con un enfoque más especializado. En 1995 salió la $2^{\text {a }}$ edición, sin apenas variaciones significativas.

Cuando redactaba el anterior estudio, Artigas venía trabajando en una serie de artículos que fraguaron en La mente del universo (Eunsa, 1999) gracias a una ayuda de la Fundación Templeton. El libro cuenta con el prólogo del cardenal Paul Poupard, entonces Presidente del Consejo Pontificio para la Cultura. El enfoque de Artigas es innovador: su propuesta sugiere que la cosmovisión científica actual es muy coherente con la inmanencia de Dios en el mundo; y esa inmanencia corresponde a un Dios personal que es al mismo tiempo completamente diferente al mundo. No hay riesgo de panteísmo en su propuesta, pues no se puede identificar ninguna criatura ni el conjunto de todas ellas con el Creador. El poder, el orden y la belleza del universo han provocado la admiración de los hombres, una actitud filosófica pero también religiosa: se reconoce algo divino en el cosmos. Hay orden y racionalidad en el universo, y esto permite levantar puentes entre la ciencia y la teología, si la ciencia se abre a unas perspectivas más amplias. En este sentido, Artigas analiza tres grupos de presupuestos para hacer ciencia: el ontológico, el epistemológico y el ético. Estos presupuestos no son científicos, aunque sean estrictamente racionales. Por lo demás, reconocer la presencia de estos presupuestos es un eficaz antídoto contra el reduccionismo cientificista que cierra el paso a todo diálogo con la trascendencia religiosa. La mente del universo contó en poco tiempo con dos ediciones tanto en castellano como en inglés $(2000,2001)$. 


\section{Estudios sobre Filosofía de la ciencia}

En 1979 Mariano Artigas defiende su tesis doctoral en Filosofía por la Universidad de Barcelona, con un trabajo titulado La confiabilidad de la ciencia y su impacto filosófico. Comienza así a adentrarse en uno de sus temas preferidos: la epistemología contemporánea y su validez. El mismo año publica el primer libro sobre este tema: Karl Popper: Búsqueda sin término (Emesa, Madrid). El estudio se publica en la colección "Crítica filosófica" que se propone exponer críticamente las principales obras filosóficas que de una manera u otra han marcado el pensamiento contemporáneo. El libro se presenta como una autobiografía intelectual de Karl Popper, uno de los filósofos más relevantes de la segunda mitad del siglo XX. A lo largo de la exposición se penetra en la vida y pensamiento del filósofo vienés, con una trayectoria vital comprometida no solo con la filosofía sino también con la acción social. Defensor de la democracia y crítico con todo totalitarismo y dogmatismo (especialmente del marxismo), Popper ha pasado a ser uno de los iconos de la cultura europea. Artigas se acerca a su biografía con una distancia crítica, pero respetuosa, mostrando la validez de su actitud antidogmática, pero indicando la parcialidad de sus conclusiones por partir de unos presupuestos cientificistas. Años más tarde, en 1995, apareció la $2^{\text {a }}$ edición sin revisiones reseñables.

Artigas siguió de cerca la obra de Popper reconociendo el valor de sus propuestas, pero sin obviar sus problemas ni compartir sus presupuestos epistemológicos. La admiración por la epistemología popperiana le llevó a comenzar una relación epistolar con el filósofo austriaco que prometía ser fecunda, pero que desgraciadamente se truncó, como él mismo explica (Artigas 2001a, 9-12). El interés por Popper le llevó a publicar dos textos inéditos del filósofo austriaco que le sirvieron para completar su interpretación. Se trata de un diálogo entre Popper y su discípulo Bartley acerca de la ética, que para Artigas supone una nueva luz para interpretar la filosofía popperiana en su conjunto. Este es el origen de un breve y muy sugerente libro titulado Lógica y ética en Karl Popper que apareció en 1998 (2a edición 2001a). Un año después aparece la versión inglesa: The Ethical Nature of 
Karl Popper's Theory of Knowledge (Berne: Peter Lang, 1999). En definitiva, lo que Artigas propone es una interpretación de la epistemología de Popper partiendo de su compromiso ético y social, sin el cual, sus propuestas científicas perderían gran parte de su calado filosófico. Por lo demás, la ética de Popper descansa en unos presupuestos realistas que, interpretados en un marco más amplio, pueden ser compartidos por la filosofía clásica: la interpretación de Artigas sobre la filosofía de Popper merece un estudio más detenido que en parte ya ha sido abordado (Corcó 1995).

En 1989 apareció un estudio sistemático sobre la epistemología contemporánea, donde Artigas volcó su reflexión personal y la docencia de muchos años en la Universidad de Barcelona: Filosofía de la ciencia experimental. La objetividad y la verdad en las ciencias (Eunsa). A diferencia del manual de "Iniciación filosófica” sobre filosofía de la ciencia, aquí no se propone presentar una exposición “académica” de esta disciplina, sino incidir en dos aspectos fundamentales de la actividad científica: la objetividad y la verdad. Este libro de Artigas supone una profundización sistemática de gran alcance prácticamente inédita hasta ese momento. Su propuesta filosófica se presenta como una alternativa razonable frente a posturas instrumentalistas o escépticas que no pocas veces se encuentran en la base de algunos planteamientos. En última instancia - afirma Artigas - la actividad científica es una práctica humana en donde se presupone el realismo filosófico (existe una realidad que puedo conocer de modo verdadero), que no parece compatible con actitudes idealistas, escépticas o relativistas. La validez de la propuesta de Artigas se ve reflejada en las sucesivas ediciones del libro a lo largo de los años $(1992,1999)$ y una traducción al inglés (2006. Knowing Things for Sure. Science and Truth, Washington: University Press of America).

La aproximación de Artigas a la epistemología se completaría con dos libros sobre autores representativos. El primero de ellos es de 1994 y llevaba por título El desafío de la racionalidad. Se trata de una reelaboración de los materiales utilizados en un curso de doctorado impartido en la Universidad de Barcelona. La exposición se centra en la filosofía de la ciencia y su relación con la racionalidad humana, al hilo de las propuestas del Círculo de Viena, Popper, Kuhn, Feyerabend, Lakatos, Stegmüller, Toulmin y Bunge. 
En el neopositivismo, la ciencia experimental se presenta a sí misma como modelo de racionalidad; pero pronto se advierte que el criterio empirista de significación, enarbolado por el positivismo lógico como modelo de racionalidad, entra en crisis porque ese criterio de verificación no resulta a su vez verificable. Por eso, la epistemología contemporánea deriva hacia el falsacionismo (Popper), el sociologismo (Khun) y en el caos epistemológico (Feyerabend). Cada vez más se llega a reconocer que la pretendida racionalidad científica descansa en factores irracionales, hasta acabar renunciándose al ideal mismo de racionalidad. Lo que propone Artigas es precisamente recuperar el valor de la racionalidad científica: la experimentación controlada de fenómenos aporta información verdadera que sirve para formular teorías científicas verdaderas: pero esa verdad científica es una verdad parcial, o como le gusta decir a Artigas, “contextual”. En la ciencia hay verdad alcanzada racionalmente; pero la racionalidad científica se debe abrir a otros tipos de racionalidad que contribuyen a formar una imagen más completa y ajustada de la realidad. El desafío de la racionalidad es una aproximación crítica - equilibrada y realista- a la epistemología del siglo XX, que merece una atenta reflexión. Pocos años más tarde (1999), vio la luz la $2^{\text {a }}$ edición en castellano.

Finalmente, uno de los últimos libros de Mariano Artigas, en colaboración con Karl Giberson es Oracles of Science. Celebrity Scientists versus God and Religion, publicado en la prestigiosa editorial Oxford University Press (2007). Como el trabajo anterior, expone el pensamiento científico de algunos autores contemporáneos: los biólogos Gould, Dawkins y Wilson y los físicos Sagan, Hawking y Weinberg. En este caso se trata más bien de seis divulgadores que han ejercido una notable influencia en la percepción que millones de personas se han forjado sobre la ciencia y sus relaciones con la religión. Algunos de estos autores ya habían sido objeto de diversos artículos divulgativos, pero ahora Artigas los analiza de manera más sistemática y profunda, menos polémica. Se deja hablar a estos autores exponiendo sus argumentos con toda su fuerza retórica; se reconocen los logros de sus planteamientos, en definitiva, se les concede el reconocimiento de ser los "oráculos" de los tiempos modernos. Una vez presentada la grandeza del 
oponente se pasa a "la valoración objetiva de pruebas y evidencias" (Arana $2008,52)$. Por eso, este libro constituye un buen ejemplo de la renovación de la apologética actual: se aboga por el rigor y la honestidad libre de prejuicios, a la hora de tratar el tema de Dios. Artigas y Giberson abordan la raíz misma de los argumentos antiteístas que suelen ir lastradas por carencias filosóficas relativas, por ejemplo, a la creación o la doctrina de la causalidad. En todo caso, esas limitaciones resultan comprensibles para un hombre de ciencia, pero no deberían ser admisibles para aquellos que se presentan como oráculos de un saber total y omnicomprensivo. Basta una mirada honesta para dejar en evidencia la aparente incompatibilidad entre ciencia y religión. Oracles of Science ha sido traducido al italiano (2010) y al castellano (2012).

\section{Estudios sobre Historia de la ciencia}

La relación aparentemente conflictiva entre ciencia y religión ha contado con dos hitos históricos fundamentales: la figura de Galileo y la recepción de evolucionismo. Para muchos "incompatibilistas" estos dos hechos son paradigmáticos de cómo la fe religiosa (y más concretamente la Iglesia Católica) han sido un obstáculo para el progreso de la ciencia. Por eso se comprende que estos dos temas hayan suscitado el interés de Mariano Artigas desde los comienzos de su actividad. El tema es ya tratado con cierto detenimiento en sus primeros libros y artículos de divulgación, pero es en los últimos años de su vida cuando se convierte en un tema de investigación histórica al más alto nivel. Para ello, buscará la colaboración con los mejores especialistas del tema.

Por lo que se refiere al caso Galileo, el detonante para comenzar esta investigación histórica más rigurosa fue su hallazgo de un nuevo documento en el Archivo de la Congregación para la Doctrina de la Fe sobre la condena de las doctrinas de Galileo. Esto sucedía en 1999, y varios estudios sobre este descubrimiento fueron publicados dos años después (Artigas 2001b; Martínez 2001; Mateo-Seco 2001). Este nuevo documento constituía solo una pequeña pieza del gran puzzle del caso Galileo, pero la tenacidad de 
Mariano Artigas por encuadrar debidamente la cuestión avivó su interés sobre el tema. Poco tiempo después coincidió en un Congreso con el profesor canadiense William R. Shea (titular de la "Cátedra Galileo" de Historia de la Ciencia de la Universidad de Padua). Hasta entonces no se conocían personalmente, pero Artigas le propuso redactar un libro conjuntamente. A pesar de lo audaz de la propuesta, Shea aceptó enseguida.

Así es como escribieron (Galileo in Rome. The Rise and Fall of an Uneasy Genius. 2003. Oxford University Press) que se dedica a analizar, con documentos archivísticos, las estancias de Galileo en Roma con motivo de las diversas sesiones con las autoridades eclesiásticas. El rigor histórico manifestado en estas páginas atempera juicios apresurados acerca del caso Galileo: no es un libro apologético, sino histórico; se exponen los hechos tal como tuvieron lugar, según se puede constatar en las fuentes documentales consultadas de manera rigurosa. Ese mismo año salió la versión castellana, con un título un poco diverso (Galileo en Roma. Crónica de 500 días). Después se sucedieron las traducciones al japonés (2005), al coreano (2006), al alemán (2006) y al italiano (2009).

El trabajo conjunto con William Shea resultó satisfactorio para ambas partes y cuajó en una honda amistad entre ellos. De nuevo emprenden un trabajo en colaboración, revisando desde una perspectiva distinta el caso Galileo. Ya no se trataba de presentar la historia tal como aconteció sino de mostrar las diversas interpretaciones que ha recibido, en el siglo XX. En efecto, en este siglo abundan las interpretaciones simplificadas o desinformaciones deliberadas que han calado en el imaginario colectivo en "el mito Galileo": el mito de la ciencia moderna frente al oscurantismo de la Iglesia. El título del libro resulta ya muy elocuente: Galileo Observed. Science and the Politics of Belief, (2006. Science History Publications); y más todavía el título de la versión castellana: El caso Galileo: mito y realidad (2009. Encuentro).

El tercer libro de la trilogía sobre Galileo tiene también una historia que merece ser reseñada. En 1981, el Papa Juan Pablo II crea una Comisión Pontificia para estudiar el papel de la Iglesia en el caso Galileo. Con este estudio, el Pontífice deseaba dar a conocer la verdad sobre la cuestión, en el contexto de la petición de perdón por los errores cometidos a lo largo 
de la historia por los hijos de la Iglesia. Once años más tarde, en 1992, se concluyen solemnemente los trabajos de la Comisión con un acto en donde intervinieron el cardenal Poupard (entonces Presidente del Pontificio Consejo de la Cultura) y el mismo Juan Pablo II. Lo que debería haber sido el cierre de un asunto espinoso se convirtió en el punto de partida de una nueva polémica: del mito Galileo se pasó al mito de la Comisión Pontificia (Sánchez de Toca 2009, 283-287). Las críticas sobre las conclusiones de la Comisión así como del método de trabajo fueron muy numerosas. Es entonces cuando Artigas propone al Subsecretario del Pontificio Consejo de la Cultura, Mons. Sánchez de Toca, trabajar conjuntamente en los Archivos para exponer de manera rigurosa y honesta los trabajos de la Comisión, con sus luces y con sus sombras. El trabajo se prolongó durante varios años, y Artigas ya no pudo ver en vida el fruto de sus investigaciones. En 2007 apareció en castellano Galileo y el Vaticano. Historia de la Comisión Pontificia de Estudio del Caso Galileo (1981-1992), (2008. BAC). La versión italiana, revisada y ampliada, vio la luz un año después, y cuenta con el presentación del Cardenal Gianfranco Ravasi (2009. Venezia: Marcianum Press).

Sobre la recepción del evolucionismo, Artigas buscó la colaboración del profesor Thomas Glick (Universidad de Boston) y de Rafael Martínez (Università della Santa Croce, Roma). La colaboración con Glick tuvo una pequeña historia, muy elocuente del talante intelectual de Artigas. Glick era uno de los mejores conocedores de la historia del evolucionismo, y en un trabajo se hacía eco de la condena que la Iglesia Católica había hecho de El origen de las especies. Artigas le escribió mostrando su extrañeza por esta afirmación, pues no le constaba que el Magisterio de la Iglesia hubiera condenado como tal la teoría evolucionista, aunque es cierto que levantó no pocas reticencias en el mundo eclesiástico. Glick le respondió citando algunas fuentes consultadas, pero mostrando desconocer el asunto de primera mano. Lo que podría haber sido una áspera polémica acabó en una sincera amistad: como resultado del intercambio epistolar, Artigas le propuso a Glick escribir juntos un libro sobre la recepción del evolucionismo en diversos autores católicos, consultando los Archivos Vaticanos. Fruto de esta investigación conjunta salió en 2006 el libro Negotiating Darwin. The Vatican confronts 
evolution 1877-1902 (The Johns Hopkins University Press). Unos años más tarde, se tradujo al castellano bajo el título Seis católicos evolucionistas: el Vaticano frente a la evolución (1877-1902) (2010. BAC). Una vez más, Artigas propone la búsqueda honesta de la verdad científica e histórica, con datos objetivos, y no la simplificación, en ocasiones interesada o ideologizada, de los problemas científicos.

\section{Conclusión}

Este rápido recorrido sobre la obra escrita de Mariano Artigas ya nos revela gran parte de su talante intelectual. Con el tiempo se valorarán detalladamente las aportaciones que llevó a cabo en los diversos campos que cultivó: filosofía de la naturaleza, epistemología e historia de la ciencia (Agazzi, 2008; Sánchez de Toca, 2008). Pero más allá de sus ideas, nuestro autor nos ha legado un estilo de trabajar intenso, riguroso, infatigable, honesto. Su modo de hacer filosofía, respetuoso con la tradición clásica cristalizada a lo largo de los siglos, no le lleva a la repetición de esquemas que solo tienen significación dentro del propio sistema escolástico, sino que busca dar respuesta a los problemas de la ciencia actual. Para que esa tradición resulte verdaderamente fecunda es necesaria una puesta al día de los principales problemas planteados por la ciencia. Su estilo de trabajar está tan alejado de un pensamiento encerrado en los estrechos cenáculos de los especialistas, como de la simplificación tendenciosa. Sus trabajos en colaboración manifiestan a un pensador que sabe trabajar en equipo, enriqueciéndose con las aportaciones de sus colegas que dominaban otras áreas del saber. El Grupo interdisciplinar de estudios sobre Ciencia, razón y fe (CRYF) es un buen ejemplo de cómo el diálogo entre académicos es posible y fecundo, cuando hay una actitud sincera de búsqueda compartida de la verdad, y no el estéril estancamiento en las propias ideas, perpetuadas de manera repetitiva en el seno de la misma escuela. Su audacia intelectual para buscar el contacto y el diálogo con los referentes reconocidos en las diversas materias le hizo conocer y tratar a filósofos de prestigio mundial, ya sea premios nobeles u hombres de Iglesia. Este estilo de hacer filosofía es quizás uno de los rasgos más atractivos de la vasta obra del profesor Artigas. 


\section{Referencias}

Agazzi, E. 2008. Aportaciones del Prof. Artigas a la epistemología y filosofía de la ciencia.” En Mariano Artigas 1938-2006. In memoriam, 61-71. Pamplona: Servicio de Publicaciones de la Universidad de Navarra.

Arana, J. 2008. “Ciencia y fe en perspectiva sistemática: los oráculos de la ciencia.” En Mariano Artigas 1938-2006. In memoriam. Pamplona: Servicio de Publicaciones de la Universidad de Navarra, 47-59.

Artigas, M. 2001a. Presentación a Lógica y ética en Karl Popper, $2^{\mathrm{a}}$ ed. Eunsa: Pamplona.

-. 2001b. “Un nuovo documento sul caso Galileo: EE 291.” Acta Philosophica 10:199-214.

Collado, S. 2008. “Mariano Artigas (1938-2006). In memoriam.” Anuario de Historia de la Iglesia 17:418-425.

Corcó, J. 1995. Novedades en el universo. La cosmovisión emergentista de Karl. $R$. Popper. Pamplona: Eunsa.

Forment, E. 1998. Historia de la filosofía en la España contemporánea. Madrid: Ediciones Encuentro.

García Cuadrado, J. A. 2007. “Mariano Artigas. In memoriam.” Scripta theologica 39:467-478.

Karol, M. 2000. Orden natural y persona humana. La singularidad y jerarquía del universo según Mariano Artigas, Pamplona: Eunsa.

López Quintás, A. 1970. Filosofía española contemporánea. Temas y autores. Madrid: BAC.

Martínez, R. 2001. “Il manoscritto ACDF, Index, Trotocolli, vol. EE, f. 291 r-v.” Acta Philosophica 10:215-242.

Mateo-Seco, L.-F., 2001. “Galileo e l'Eucaristia. La questione teológica dell'ACDF, Index, Trotocolli, EE, f. 291 r-v.” Acta Philosophica 10:243-256.

Sánchez de Toca, M. 2008. “Ciencia y fe en perspectiva histórica: estudios sobre Galileo.” En Mariano Artigas 1938-2006. In memoriam, 37-46. Pamplona: Servicio de Publicaciones de la Universidad de Navarra.

Sánchez de Toca, M. 2009. Galileo e il Vaticano. Storia della Pontificia Commisione di Studio sul Caso Galileo (1981-1992). Venezia: Marcianum Press.

Sanguineti, J. J. 2003. Prólogo a Filosofía de la naturaleza, de M. Artigas, 17-18, 4a ed. Pamplona: Eunsa. 